\section{Amélioration de la technique de dissection du tractus digestif et des glandes salivaires des glossines pour la mise en évidence des divers stades de développement des trypanosomes}

\author{
J.M.L. Kazadi ${ }^{1}$ \\ P. Elsen ${ }^{2}$ \\ M. Jochems ${ }^{1}$ \\ J. Van Hees ${ }^{1}$ \\ J. Van den Abbeele ${ }^{2}$ \\ P. Kageruka ${ }^{1}$
}

KAZADI (J.M.L.), ELSEN (P.), JOCHEMS (M.), VAN HEES (J.), VAN DEN ABBEELE (J.), KAGERUKA (P.). Amélioration de la technique de dissection du tractus digestif et des glandes salivaires des glossines pour la mise en évidence des divers stades de développement des trypanosomes. Revue Elev. Méd. vét. Pays trop., 1994,47 (1) : 89-92

Les auteurs décrivent une technique améliorée de dissection de l'intestin moyen et des glandes salivaires des glossines. En ajoutant à la dissection le proventricule, la méthode permet d'obtenir un tableau plus complet du développement des trypanosomes chez la mouche infectée.

Mots clés: Glossina - Glande salivaire - Proventricule - Intestin - Trypanosoma.

\section{Introduction}

Chaque stade de développement du trypanosome occupe une portion anatomique précise du vecteur et semble y être intimement adapté, ces adaptations successives jouant leur rôle respectif dans l'évolution du développement du parasite (11). Certains de ces stades, comme celui des formes mésocycliques, sont mal connus et l'on ignore leurs rôles dans la dynamique de la transmission cyclique. La mise en évidence de stades précis du développement des trypanosomes chez la glossine fait appel à des techniques de dissection de plus en plus fines.

Quoique controversées $(6,7)$, plusieurs techniques de dissection ont été utilisées pour mettre en évidence les trypanosomes chez la glossine $(1,3,5,8,9,13)$. Elles sont laborieuses et incomplètes en ce sens qu'elles ne se limitent souvent qu'à l'extraction des glandes salivaires et/ou d'une portion du tube digestif, le proventricule restant généralement inexploré. Pourtant, d'après les travaux de STEIGER (10), cet organe semble être le siège des formes mésocycliques, une des étapes de la chaîne de la métacyclogenèse.

1. Institut de Médecine tropicale Prince Léopold, Département de Santé Animale, Nationalestraat 155, B-2000 Antwerpen 1, Belgique.

2. Institut de Médecine tropicale Prince Léopold, Service d'Entomologie, Nationalestraat 155, B-2000 Antwerpen 1, Belgique.

Reçu le 15.10.1993, accepté le 2.3.1994.
Le but de ce travail est de décrire une technique de dissection permettant l'accès facile du proventricule afin de l'incorporer à l'examen classique du reste du tube digestif et des glandes salivaires, tout en améliorant la technique de dissection de ces organes.

\section{Rappel anatomo-physiologique}

Le proventricule fait partie du tube digestif ; il est situé au carrefour de trois organes : d'une part, le jabot pédiculé et l'œsophage qui s'abouchent en un orifice commun à sa face ventrale et d'autre part, l'intestin moyen qui $y$ pénètre par sa face dorsale (fig.1,2). Ce complexe est localisé dans l'axe médian du thorax, légèrement en avant des pattes antérieures et un peu en amont du ganglion nerveux thoracique.

Le proventricule établit une régulation à trois voies du flux de sang ingéré au moment du repas de la mouche. Son rôle physiologique n'est pas entièrement élucidé, mais il semble jouer une part importante dans le processus de la métacyclogenèse, notamment dans la sécrétion de la membrane péritrophique (12) et dans le passage des formes mésocycliques vers les glandes salivaires (11).

En coupe sagittale, cette entité est une formation complexe qui se présente comme une invagination en forme de champignon de la portion antérieure du tube digestif dans l'intestin moyen, dont la partie antérieure, recouverte par l'invagination précédente, est épaissie pour former le bourrelet annulaire (2). De part et d'autre du tractus digestif cheminent deux glandes salivaires. Ces glandes d'aspect crénelé irrégulier et transparent, se présentent sous une forme tubuleuse et contournée d'environ 30 $\mathrm{mm}$ de long, soit deux fois la taille d'une mouche adulte; elles s'étendent de la base de l'hypopharynx jusque dans l'abdomen où elles sont nettement plus épaisses.

\section{Technique de la dissection par voie postérieure}

La dissection doit être pratiquée sur des mouches non gorgées et fraîchement tuées après un séjour de 5 minutes en atmosphère d'azote ou en glacière. Les outils de dissection doivent être suffisamment désinfectés, par exemple dans l'alcool (70 p.100) - hibitane (0,5 p.100) pour éviter le risque de contamination des organes disséqués des mouches infectées de trypanosomes avec ceux des mouches saines. Aucune pression ne doit être exercée sur le thorax pour ne pas entraîner le déplacement éventuel du proventricule. La dissection s'effectue en trois étapes distinctes. Toutes ces étapes sont exécutées sous une loupe binoculaire (x 160), tandis que les préparations sont observées au microscope (x 400). 


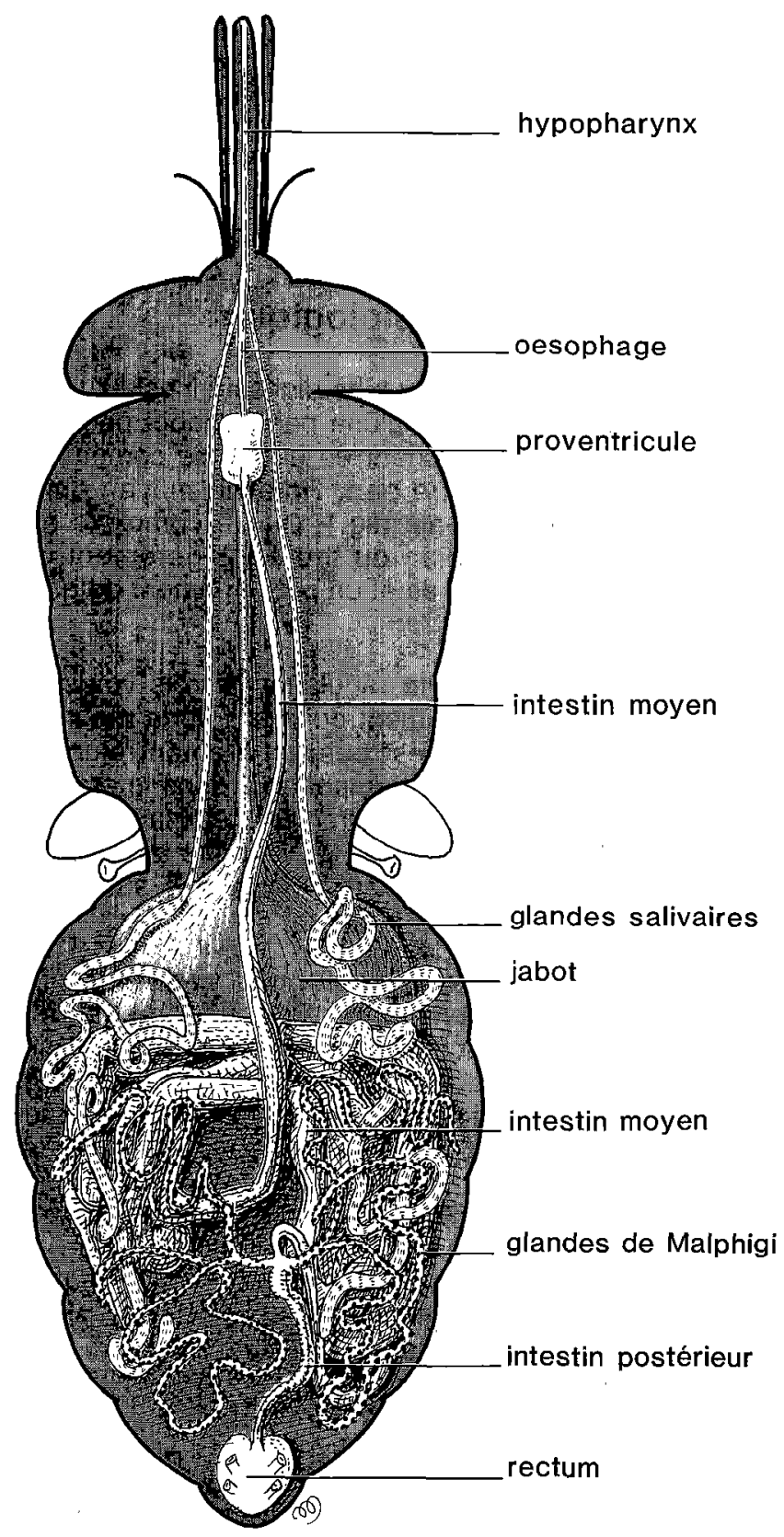

Figure 1: Vue dorsale du tractus digestif d'une glossine.

\section{Extraction abdominale}

Saisir la mouche avec une pincette courbe, la déposer en position de décubitus dorsal sur une lame porte-objet pourvue d'une grosse goutte d'eau physiologique. D'une main, maintenir la mouche au moyen d'une aiguille montée courbe en plaçant celle-ci entre les coxae des pattes métathoraciques. De l'autre main, pratiquer avec un bistouri fin une incision postéro-médio-ventrale de l'abdomen intéressant le $7 e$ sternite de la mouche. Chez le

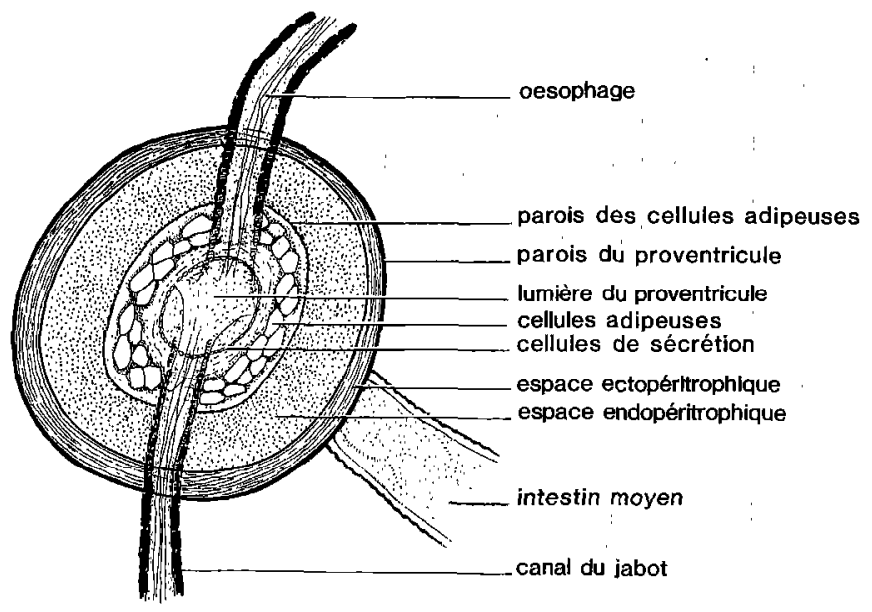

Figure 2 : Vue ventrale d'un proventricule monté.

mâle, cette incision traverse l'hypopigium. Ensuite, tout en maintenant la glossine avec l'aiguille courbe, introduire une pincette courbe au niveau de l'incision, en 'écarter les bords et saisir, à hauteur du renflement de l'ampoule rectale, l'extrémité postérieure du tractus digestif (fig:3).

Par un mouvement de traction horizontale et régulière, retirer délicatement vers l'extérieur tout le contenu de la cavité abdominale que l'on dépose sur une lame porteobjet pourvue d'une goutte d'eau physiologique. Les glandes salivaires restent souvent entremêlées à la masse musculo-adipeuse du plancher dorsal. Il faut donc les extraire par une traction continue et les observer au microscope entre lame et lamelle. Ces glandes ont tendance à se rétracter rapidement à l'air libre. II arrive néanmoins, surtout chez les individus ténéraux, que les glandes salivaires se recroquevillent dans les angles antéro-latéraux de l'abdomen (8). Dans ce cas, il faut passer à l'étape suivante.

\section{Extraction des glandes salivaires}

La deuxième phase consiste à extraire aussi soigneusement que possible les deux glandes salivaires. Introduire la pincette courbe, vers le bas, de chaque côté de l'incision et prélever, à tour de rôle, les glandes salivaires gauche et droite qui se rompent au niveau de la base du proboscis.

\section{Prélèvement du proventricule}

La troisième phase vise l'extirpation du proventricule ; retirer l'aiguille courbe implantée entre les coxae des pattes métathoraciques, appuyer l'index sur le ventre vidé de la glossine et pratiquer une section transversale, un peu en arrière des coxae des pattes prothoraciques. 
ENTOMOLOGIE

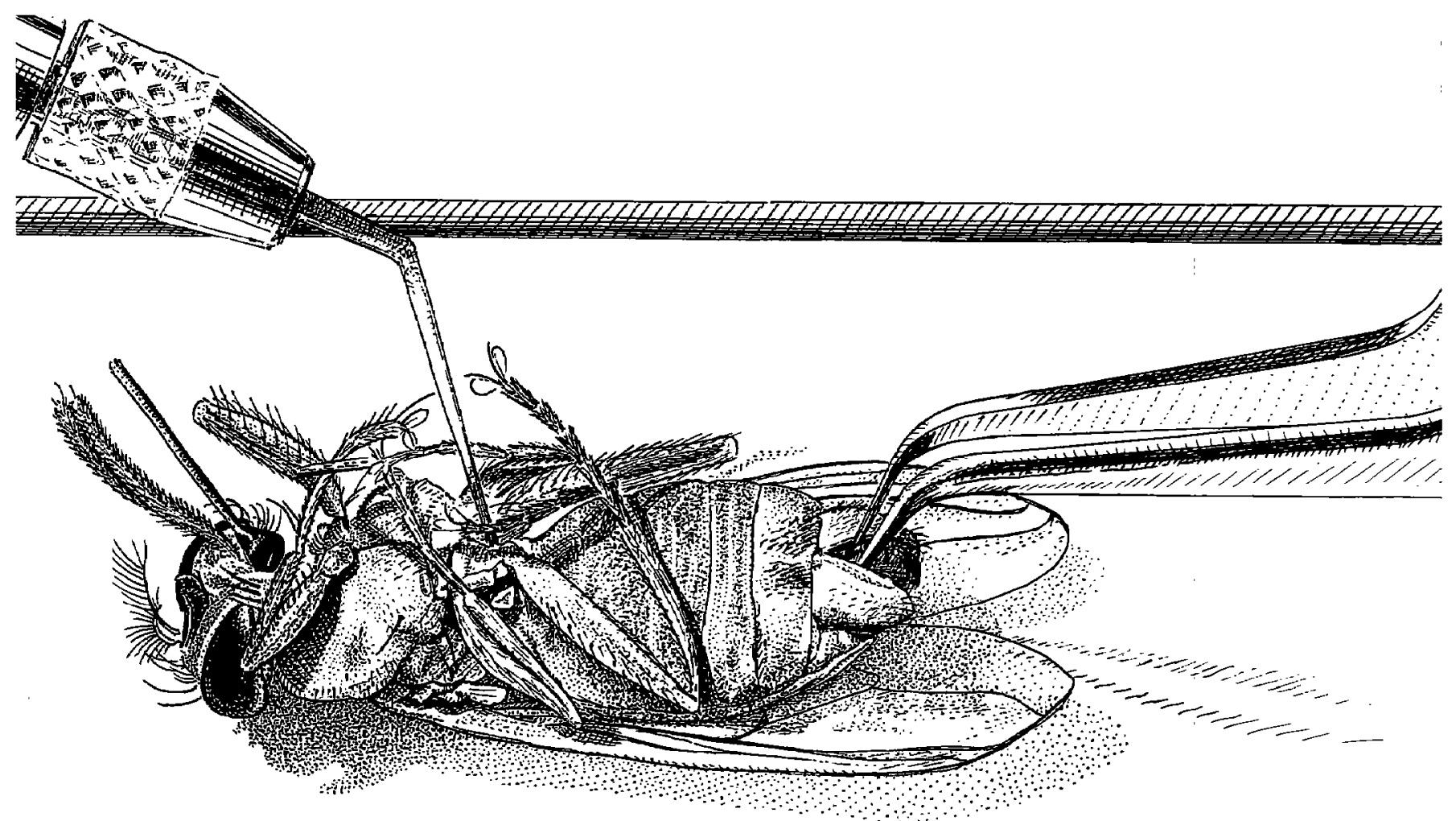

Figure 3 : Extirpation de l'intestin moyen et des glandes salivaires à partir de l'extrémité postérieure.

Déposer la portion céphalo-thoracique en position de décubitus dorsal sur une lame porte-objet pourvue d'une goutte d'eau physiologique. Ensuite, l'opérateur fixe avec l'aiguille courbe la base du bulbe du proboscis et introduit la pincette droite un peu vers le haut et en avant de la partie médiane de ladite portion pour saisir et ensuite extirper le proventricule (fig.4). Celui-ci est souvent accompagné des trois moignons formés par le canal de l'oesophage, du jabot et de l'intestin moyen (fig.2).

Afin d'évaluer au microscope (x 400) la présence de trypanosomes au niveau de différentes localisations anatomiques, on conseille de déposer, sur une seule lame préalablement pourvue de trois gouttes d'eau physiologique, les organes disséqués dans l'ordre suivant : intestin moyen, proventricule et glandes salivaires, chacun étant placé sous une lamelle couvre-objet distincte.

\section{Conclusions}

La dissection par voie postérieure offre les avantages suivants :

- elle est facile et rapide ; il n'est pas nécessaire d'enlever les pattes ni les ailes ;

- elle permet de réaliser avec soin l'extraction du tube digestif, de l'anus au proventricule, l'extirpation complète des deux glandes salivaires, des gonades, y compris éventuellement les glandes annexes;

- elle permet de réaliser aisément l'extraction du proventricule en préservant sa structure anatomique, offrant la possibilité de démontrer son intérêt dans la métacyclogenèse.

Dans un travail ultérieur, nous montrerons dans quelle mesure la prise en compte de la présence des formes proventriculaires contribue à l'amélioration de la formule de la capacité vectorielle intrinsèque établie par LE RAY (3). 


\section{Communication}

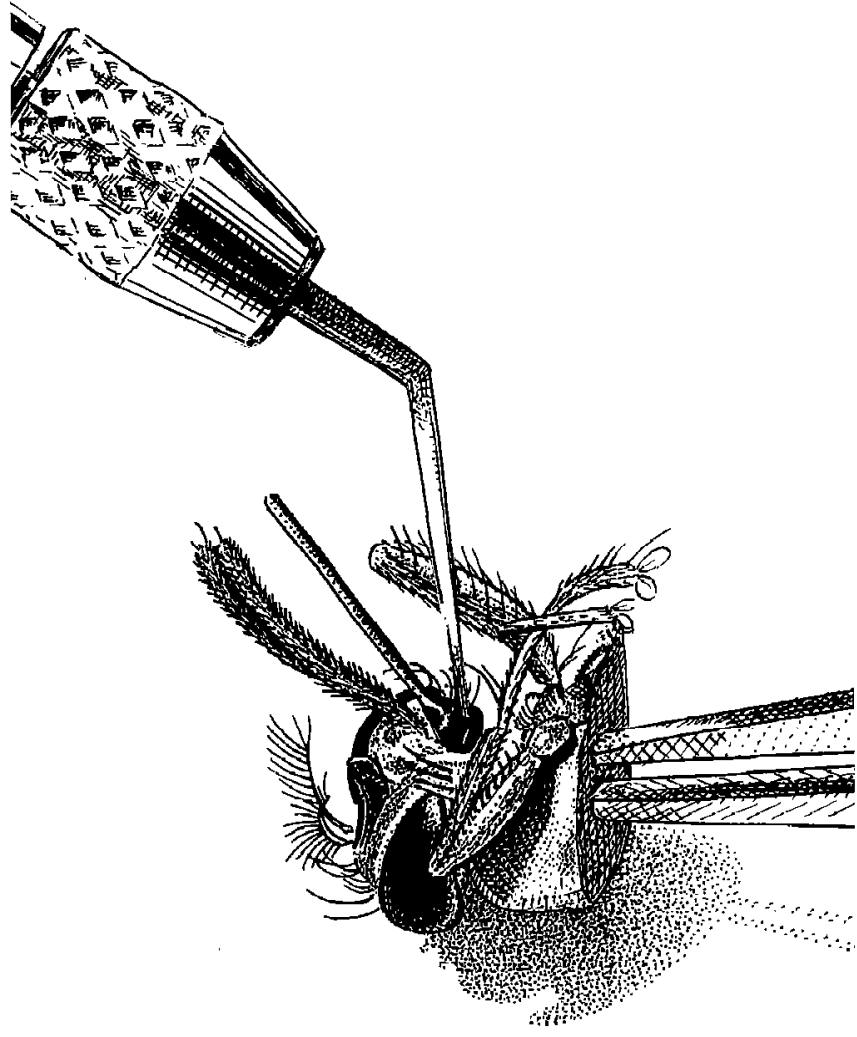

Higure 4 : Position de l'aiguille courbe et de la pincette droite pour extirper le proventricule.

\section{Remerciements}

Ce travail bénéficie du support financier de l'Administration générale à la Coopération et au Dévèloppement (gouvernement belge). Les auteurs remercient $\mathrm{M}$. $\mathrm{H}$. VANVINCKENROYE pour les soins apportés à la réalisation des dessins ainsi que $\mathrm{Mme} \mathrm{Cl}$. MATTELAERE pour son aide.

\section{Bibliographie}

1. EVANS (D.A.), ELLIS (D.S.). Recent observations on the behaviour of certain trypanosomes within their insect hosts. Adv. Parasitol, 1983, 22: $1-42$.

2. ITARD (J.). Les glossincs ou mouches tsé-tsé. Maisons $\Lambda$ lfort, IEMVT, 1986. (Etudes et Synthèses de l'IEMVT n ${ }^{\circ} 15$ ).

3. JEHFERIES (D.), HELFRICH (M.P.), MOLYNEUX (D.H.). Cibarial infections of Trypanosoma vivax and $T$. congolense in Glossina. Parasitol. Res., 1987, 73: 289-292.
4. LL RAY (D.). Vector susceptibility to african trypanosomes. Annls. Soc. belge Méd. trop., 1989, 69 (suppl. 1) : 165-171.

5. LLOYD (LL), JOHNSON (W B ). The trypanosome infections of tsetse-flies in northern Nigeria and a new method of estimation. Bull. ent. Res., 1924, 14: 265-288.

6. MEHLITZ (D.), TIETJEN (U.). Trypanosome infection rates in tsetse midguts using a short-term in vitro culture technique. Acta trop., 1988, 45: 183-184.

7. OTIENO (L.H.). Inadequacy of the dissection method of estimating trypanosome infection rates. Ann. trop. Med. Parasit.1983, 77 (3): 329-330.

8. PENCHENIER (L.), ITARD (J.). Une houvelle technique de dissection rapide des glandes salivaires et de l'intestin des glossines. Cah. ORSTOM., Sér. Enu. méd. Parasit., 1981, 19 (1): 55-57.

9. POLLOCK (J.N.). Manuel de lutte contre la mouche tsé-tsé. Rome, FAO, 1982

10. STEIGER (R.F.). On the ultrastructure of Trypanosoma (Trypanozoon) bruce $i$ in the course of its life and some related aspects. Acta trop., 1973, $30(1-2): 65-167$

11. VICKERMAN (K.), TETLEY (L.), HENDRY (K.A.K.), TURNER (C.M.R.). Biology of african trypanosomes in the tsetse fly. Biology of the Cell., 1988, 64 : 109-119.

12. WIGGLESWORTH (V.B.). Digestion in the tsetse-fly : A study of structure and function. Parasitology, 1929, 21: 288-321.

13. WILLET (K.C.). A special method for the dissection of Glossina. Ann. trop. Med. Parasit., 1955, 49: 376-383.

KAZADI (J.M.L.), ELSEN (P.), JOCHEMS (M.), VAN HEES (J.) VAN DEN ABBELLE (J.), KAGERUKA (P.). Improvement of the technique of mid-gut and salivary gland dissection in tsetse fly for determining the different stages of trypanosome development. Revue Élev. Méd. vét. Pays trop., 1994, 47 (1) : 89-92

An improved technique of mid-gut and salivary gland dissection in tsetse fly is described. The incorporation of the proventriculus in the dissection procedure gives a far better picture of trypanosome development in the infected fly.

Key words: Glossina -Salivary gland - Proventriculus - Intestine - Trypanosoma. 